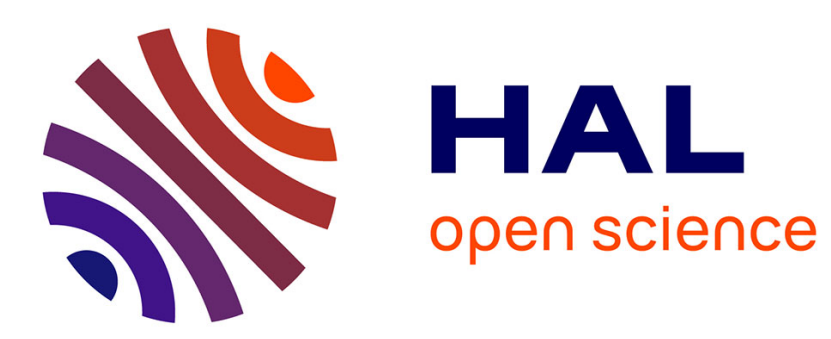

\title{
Properties of unipolar magnetic field pulse trains generated by lightning discharges
}

Ivana Kolma Sová, Ondřej Santolík

\section{To cite this version:}

Ivana Kolma Sová, Ondřej Santolík. Properties of unipolar magnetic field pulse trains generated by lightning discharges. Geophysical Research Letters, 2013, 40 (8), pp.1637 - 1641. 10.1002/grl.50366 . insu-01396166

\section{HAL Id: insu-01396166 https://hal-insu.archives-ouvertes.fr/insu-01396166}

Submitted on 14 Nov 2016

HAL is a multi-disciplinary open access archive for the deposit and dissemination of scientific research documents, whether they are published or not. The documents may come from teaching and research institutions in France or abroad, or from public or private research centers.
L'archive ouverte pluridisciplinaire HAL, est destinée au dépôt et à la diffusion de documents scientifiques de niveau recherche, publiés ou non, émanant des établissements d'enseignement et de recherche français ou étrangers, des laboratoires publics ou privés. 


\title{
Properties of unipolar magnetic field pulse trains generated by lightning discharges
}

\author{
Ivana Kolmašová ${ }^{1}$ and Ondřej Santolík ${ }^{1,2}$ \\ Received 1 February 2013; revised 13 March 2013; accepted 13 March 2013; published 23 April 2013.
}

[1] We analyze and describe trains of regular unipolar microsecond-scale magnetic field pulses produced by intracloud lightning discharges. Waveforms of the magnetic field are measured using a newly developed broadband analyzer with a sampling interval of $12.5 \mathrm{~ns}$. The observed trains contained several tens of pulses, and the time interval between neighboring pulses typically varied between 1 and $10 \mu \mathrm{s}$. The amplitude of the pulses also varied by 1 order of magnitude. A systematic analysis of these variations is done for the first time. The interpulse interval is usually increasing (on average by $4.1 \mu \mathrm{s}$ during a train), and the pulse amplitude is decreasing (on average by $15 \%$ of the maximum value within a given train). We propose a possible generation mechanism based on a hypothesis that periodical charge structures are present in the thundercloud. This mechanism can explain the observed evolution of peak amplitudes and interpulse intervals. Citation: Kolmašová, I., and O. Santolík (2013), Properties of unipolar magnetic field pulse trains generated by lightning discharges, Geophys. Res. Lett., 40, 1637-1641, doi:10.1002/grl.50366.

\section{Introduction}

[2] Trains of electric field and magnetic field pulses were found to be connected with various lightning phenomena. Preliminary breakdown pulses [Nag and Rakov, 2008], stepped leader pulses [Lee et al., 2006], dart-stepped leader pulses [Davis, 1999], chaotic leader pulses [Gomes et al., 2004], pulses produced by bouncing-wave-type lightning discharges [Nag and Rakov, 2009], and bursts associated with either $K$ changes or $M$ components were observed [Rakov et al., 1992, 1996]. The shapes of individual types of pulses are generally similar, but the lengths of the rising and falling edges, the widths of the pulses, and the interpulse intervals vary from microsecond to millisecond scales. These transient events may play an important role in the complexity of the atmospheric electromagnetic fields [Füllekrug and Fraser-Smith, 2011].

[3] The trains of pulses occurring in cloud flashes were first studied by Krider et al. [1975], who compared the time intervals between neighboring pulses and the interval between bright steps in dart-stepped leader photographed,

\footnotetext{
${ }^{1}$ Institute of Atmospheric Physics, Academy of Sciences of the Czech Republic, Prague, Czech Republic.

${ }^{2}$ Faculty of Mathematics and Physics, Charles University, Prague, Czech Republic.

Corresponding author: I. Kolmašová, Institute of Atmospheric Physics, Academy of Sciences of the Czech Republic, Boční II 1401, 14131 Prague 4, Czech Republic. (iko@ufa.cas.cz)

(C)2013. American Geophysical Union. All Rights Reserved. 0094-8276/13/10.1002/grl.50366
}

and reported by Schonland [1956]. Based on the similarity of these time intervals, Krider et al. [1975] suggested that a dart-like $K$ change developing in a stepped manner could produce the observed uniform pulses. Similar trains in electric field waveforms of cloud-to-ground and intracloud lightning discharges were observed and reported by Rakov et al. [1992, 1996]. According to these studies, the movement of charge in the existing intracloud lightning channel between the strokes can give rise to the microsecond-scale electromagnetic pulses. The pulses occurring during the $K$ step and $M$ hook field changes differed in terms of amplitudes, regularity, and intervals between neighboring pulse trains. Rakov et al. [1992] found that 53\% of the recorded $M$ components and only $24 \%$ of the recorded $K$ changes were accompanied by the microsecond-scale pulse activity.

[4] Krider et al. [1975] measured the time interval between successive pulses in both maritime (Florida) and continental (Arizona) conditions. The mean values of interpulse intervals were similar: $6.1 \mu$ s for the seaside and $5.1 \mu \mathrm{s}$ for the inland, but the distribution of interpulse intervals for continental conditions was narrower than the distribution for maritime conditions. Thanks to to the multipoint measurement (five stations), Davis [1999] was able to estimate locations and speeds of the movement of the leaders from his measurements of the time derivative of the electric field. Due to a short length of the waveform records $(205 \mu \mathrm{s}$ only), he limited his study to the interpulse interval. The obtained averages of the interpulse intervals were $2.8,7.6$, and $5.1 \mu \mathrm{s}$ for trains in dart-stepped leaders, in leaders preceding new ground termination, and for trains in intracloud (IC) discharges, respectively.

[5] The amplitude of the pulses and the spacing between successive pulses are changing with time within a given train. Rakov et al. [1992, 1996] reported that the amplitudes of the pulses and the pulse repetition rates tended to decrease with time. Davis [1999] not only showed cases of growing interpulse intervals in both dart-stepped leaders and IC leaders but also showed examples of leaders preceding new ground termination which did not exhibit any change of the interpulse interval. The growing intervals were consistent with his measurements of a decreasing speed of the leader movement assuming that the length of the steps in the propagation of the leaders remained unchanged. This assumption was based on optical observations of Schonland [1956], who reported approximately equidistant steps of about $10 \mathrm{~m}$. Wang et al. [2010] classified four types of bursts according to the evolution of the interpulse interval and the polarity of the pulses and assigned them to different lightning phenomena. The pulses belonging to a given train usually have the same polarity. Rakov et al. [1996] reported that the trains with negative and positive pulses occurred with the same probability. They also noted occasional reversals of the polarity of pulses 


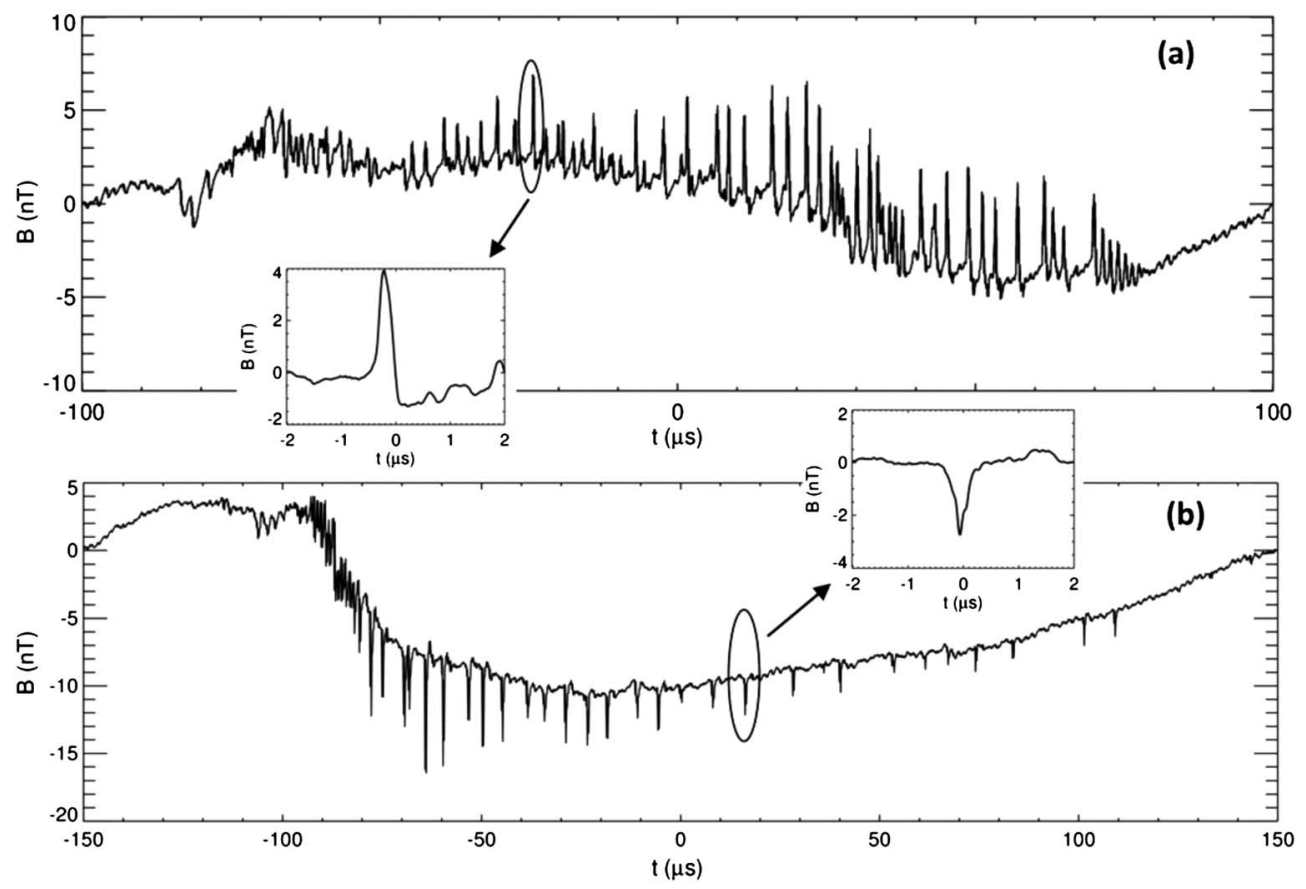

Figure 1. Examples of the trains with (a) positive and (b) negative pulse polarities. Detailed examples of individual pulses are shown in the insets.

within some of the observed trains. Davis [1999] found that for $66 \%$ of pulses, the polarity was consistent with the direction of the current flowing in the channel. The reversal in the polarity was attributed to changes of the channel geometry and to a possible development of branches.

[6] In the present study, we analyze properties of the trains of regular unipolar microsecond-scale magnetic field pulses produced by intracloud lightning phenomena in continental conditions (Prague, Czech Republic). We use a new broadband analyzer with a sampling interval of $12.5 \mathrm{~ns}$. Our time resolution is by more than 1 order of magnitude better than the limit of measuring systems used in studies of Krider et al., 1975] and Rakov et al., 1996]. Our time resolution is also 4 times better than the resolution of Davis [1999], and the length of our recordings is about 3 orders of magnitude larger than in his data. Our measurements are therefore suitable for a clear identification of individual microsecondscale pulses in the trains and, at the same time, for long recordings of multiple trains in a sequence.

[7] In section 2, we describe our experimental setup, and in section 3, we summarize our results concerning the time intervals between the trains, their lengths, the number of pulses in the individual trains, and the interpulse time intervals. In section 4 , we present a systematic analysis of the evolution of interpulse intervals and pulse amplitudes within the trains, and, finally, in section 5, we discuss our results.

\section{Instrumentation}

[8] For our measurements, we use a magnetic field antenna coupled with a ground-based version of a broadband high-frequency analyzer which is being developed for the TARANIS spacecraft (http://babeta.ufa.cas.cz/TARANIS; http://smsc.cnes.fr/TARANIS). The analog part of the analyzer includes two fully differential amplifiers, two antialiasing filters, and a set of 12 band-pass filters with amplifiers and RMS detectors. The signals from these detectors are used as input data for a flexible event detection algorithm. The core of the digital part of the analyzer is a field-programmable gate array, where the sampled and digitized signals are processed. The analyzed frequency band goes from $5 \mathrm{kHz}$ to $37 \mathrm{MHz}$. The signal is sampled at $80 \mathrm{MHz}$, and parts of the waveforms are recorded based on the results of the event detection algorithm. This procedure provides us with $120 \mathrm{~ms}$ long waveforms every $5 \mathrm{~min}$. The time assignment is done by a GPS receiver connected to the analyzer. The magnetic field antenna is formed by a single loop of a $50 \Omega$ coaxial cable with a loop diameter of $1 \mathrm{~m}$ (a similar antenna system was used by Krider and Noggle [1974]). We approximate the voltage induced in the antenna loop by the product of the loop area and the magnetic field time derivative $\mathrm{d} B / \mathrm{d} t$ in the direction perpendicular to the loop. We numerically integrate the waveform records to estimate the $B$ component in this direction. The integrated noise of the analyzer is about $0.14 \mathrm{nT}$ in the frequency range from $1 \mathrm{MHz}$ (the reciprocal value of the typical pulse time scale) to $37 \mathrm{MHz}$ (upper frequency limit of our measurement).

\section{Properties of the Pulse Trains}

[9] Our original data set consisted of 1409 regular pulses in 33 trains recorded during two thunderstorms in summer 2011 in Prague, Czech Republic. We do not have reliable information about the distances to the lightning phenomena that generated the recorded pulses. However, based on the most distant return strokes recorded by the Central European Lightning Detection Network (P. Novák, private communication, 2011) and observed during the same campaign, we can estimate that the distances to the sources of the observed high-frequency signals were probably less than $20 \mathrm{~km}$.

[10] The amplitudes of all the pulses and the times of their peak values were estimated from the integrated $B$ waveforms. To record a pulse, we have chosen a threshold of 



Figure 2. (a)Time interval between the neighboring trains. (b) Duration of the trains. (c) Number of pulses in the trains. (d) Time interval between neighboring pulses in each train. (e) Ratio of the largest to the smallest amplitude of pulses in each individual train. (f) Pulse amplitudes normalized by their maximum in each individual train.

$1 \mathrm{nT}$ for the peak amplitude. This threshold is several times larger than the noise level of the analyzer. Each individual pulse is considered to be unipolar if the immediately following overshoot of the opposite polarity does not exceed one half of the peak amplitude of the original pulse. A group of pulses is considered to form an individual train when the time interval between the last pulse of a train and the first pulse of the next train exceeds $80 \mu$ s. This threshold has been chosen to be more than 1 order of magnitude higher than the expected interpulse interval (several microseconds). Examples of waveforms of individual pulses and trains with positive and negative pulse polarities are shown in Figures 1a and $1 \mathrm{~b}$, respectively.

[11] Out of the original data set of 33 trains, we have observed 2 isolated trains, each in a separate $120 \mathrm{~ms}$ long recording, and 31 trains grouped in three separate sequences (with 12, 13, and 6 trains, respectively). We have measured the time interval between the beginnings (times of the first pulses) of the neighboring trains in these sequences. The histogram of these time intervals is plotted in Figure 2a. The distribution clearly has a "heavy tail" and is far from being normal. The geometrical average of the obtained values is $5.6 \mathrm{~ms}$. The last train in the shortest sequence has a significantly longer time delay ( $68 \mathrm{~ms})$.

[12] As the next step of our analysis, all trains which contained bipolar pulses and trains with both pulse polarities have been excluded from our statistics. Out of the 25 remaining trains, 8 trains contain pulses with positive polarities, and 17 trains have negative pulse polarities, corresponding to relations between directions of distant source currents and their unknown positions with respect to the orientation and position of the antenna. This analyzed data set contains the total number of 967 individual pulses. The duration of the individual trains varies from 48 to $448 \mu \mathrm{s}$ with a mean value of $176 \mu$ s (Figure $2 \mathrm{~b}$ ). This is more than 30 times shorter than the typical time intervals between the trains. The total number of pulses in a train varies from 12 to 109 with a mean value of 39 (Figure 2c). The time interval between neighboring pulses within the trains ranges between 0.7 and $28 \mu \mathrm{s}$ with a mean value of $4.7 \mu \mathrm{s}$ (Figure 2d). The ratio between the smallest and the largest amplitude of pulses in each individual train varies from 0.1 to 0.4 with a mean value of about 0.2 (Figure 2e). The pulse amplitudes follow a wide distribution (Figure 2f), but on average, they reach approximately 0.5 of their maximum in a given train.

\section{Evolution of the Pulse Amplitude and of the Interpulse Interval Within the Trains}

[13] Figures 3a and 3b, respectively, show an example of evolution of the interpulse interval and the pulse amplitude 

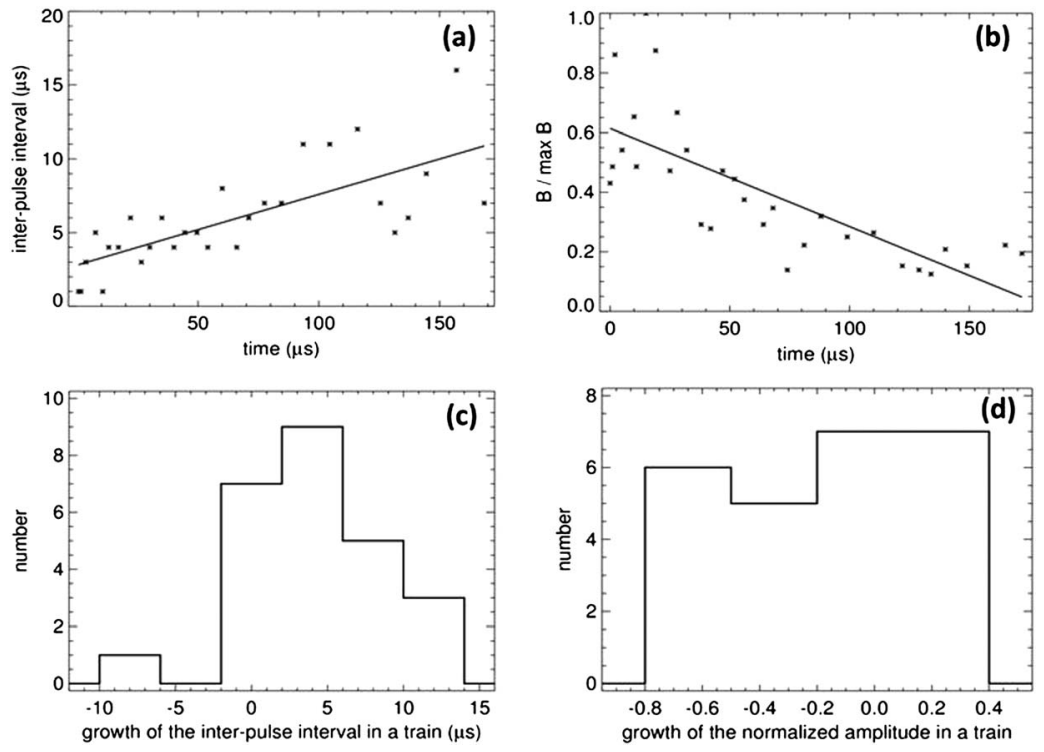

Figure 3. Evolution of (a) the interpulse interval and (b) the pulse amplitude normalized by its maximum value in a train from Figure 1b. Solid lines show linear fits. Histograms of linear trends for all analyzed trains: (c) The growth of the interpulse interval within each individual train. (d) The amplitude growth related to the maximum pulse amplitude in each train.

normalized by its maximum within the train from Figure $1 \mathrm{~b}$. The fluctuations of the obtained results are significant, but the general trend is that the interpulse interval is increasing and the pulse amplitude is decreasing within this train. To roughly characterize the evolution of the interpulse intervals and normalized pulse amplitudes, we have estimated linear trends in all trains. We have calculated coefficients of a linear least squares regression as a function of time. An example of the regression line is overplotted in Figures 3a and $3 \mathrm{~b}$. The histograms of the growth/decay rates in the separate pulse trains are plotted in Figures $3 \mathrm{c}$ and $3 \mathrm{~d}$, respectively, for the interpulse interval and the amplitude. The interpulse interval rises on average by $4.1 \mu \mathrm{s}$ during the train duration, and, during the same time, the amplitude drops on average by $15 \%$ of its maximum. However, the spread of the obtained values is very large (from a decrease by $70 \%$ up to an increase by $37 \%$ ) in the case of the normalized amplitudes.

[14] Although the approximation by a linear function is very crude, it suggests that the interpulse interval is typically increasing in our data set. To verify this result, we have calculated the rank correlation coefficients between time and interpulse intervals and the corresponding probabilities of random occurrence of a positive or a negative correlation. We have further selected only those trains where this probability is less than $5 \%$, reducing the data set down to 16 trains. Out of these, 15 trains have a significantly positive correlation coefficient with an average value of 0.61 , and only one train has a negative correlation coefficient of -0.51 . For the normalized amplitude, the situation is less clear, with 6 significantly positive and 11 significantly negative correlation coefficients (average values of 0.42 and -0.66 , respectively). This analysis therefore shows that the typical evolution pattern is represented by growing interpulse intervals and decreasing normalized amplitudes. However, cases with increasing amplitudes or uncertain evolution trends also occur.

\section{Discussion}

[15] In this study, we attempt to improve our experimental knowledge of the properties of the trains of unipolar pulses. The resolution of our instrumentation is about by more than 1 order of magnitude better than the limit of measuring systems used in previously published studies [Krider et al., 1975; Rakov et al., 1996], allowing us to fully resolve individual pulses and to also measure long sequences of pulse trains.

[16] To discuss our results, we can start by comparing the interval between the trains in our data set with the time properties of the $K$ change and $M$ component sequences reported by Thottappillil et al. [1990]. According to their measurements of close lightning flashes, the geometricmean interval between neighboring $K$ changes or $M$ components, respectively, were 12.5 or $2.1 \mathrm{~ms}$. The geometric-mean time interval between neighboring trains in our records is about $6 \mathrm{~ms}$, which is 2 times shorter than the time interval between $K$ changes and 3 times longer than the time interval between $M$ components reported by Thottappillil et al. [1990]. However, our histogram of time intervals between neighboring trains (Figure 2a) is much closer to the histogram for $K$ change intervals plotted by Thottappillil et al. [1990, Figure 4].

[17] Shao et al. [1995] reported that $K$ changes are indistinguishable from dart leaders and attempted leaders in their radio interferometric observation. They concluded that, above the bottom of the thundercloud, these three lightning phenomena are identical. We can therefore also compare the time interval between neighboring trains in our records with the interstep intervals of dart-stepped leaders. Krider et al. [1977] reported that the mean interval between dart steps was $6-8 \mu \mathrm{s}$, which is in good agreement with our observations.

[18] The mean value of the interpulse interval in our data set is about $4 \mu \mathrm{s}$, which is consistent with the observations of Krider et al. [1975], who reported the mean value of $5.1 \mu \mathrm{s}$ for the time interval between successive pulses measured in Arizona. The histogram plotted in Figure $2 \mathrm{~d}$ is also 
very close to the histogram of Krider et al. [1975, Figure 3b]. We can therefore conclude that we have measured the same phenomenon as Krider et al. [1975] and [Rakov et al., 1992, 1996] and that the observed pulse trains are most probably connected with $K$ changes and dart-stepped leaders. Combining the occurrence rate $(50 \%)$ of $K$ changes in interstroke intervals reported by Thottappillil et al. [1990] with the $24 \%$ fraction of $K$ changes accompanied by the microsecond-scale activity [Rakov et al., 1992], we obtain a relatively small probability of about $12 \%$ that a latter part of an interstroke interval contains $K$-related trains of pulses. Similar result can be obtained by combining the probability of occurrence of the dart-stepped leaders in the interstroke intervals [Rakov and Uman, 2003] with the probabilities of multiple stroke lightning.

[19] In spite of this relatively low occurrence, observations of the pulse trains can bring new information about the charge structure in the thunderclouds, based on the temporal evolution of pulse properties. According to our measurements, we can confirm that the most frequent evolution pattern of pulse train is characterized by an increasing interpulse interval and a decreasing pulse amplitude within an observed train, but we have less frequently observed all combinations of the evolution of the pulse amplitude and the interpulse interval. These different evolution patterns also occurred during a single $120 \mathrm{~ms}$ long record and probably belonged to the same type of lightning process. Our results therefore disagree with the assignment of different evolution patterns to particular lightning phenomena proposed by Wang et al. [2010]. Our measurements rather indicate that evolution patterns of the peak amplitudes and interpulse intervals reflect the movement of a dart-stepped leader inside the cloud.

[20] The observed variations of the pulse amplitudes can be also tentatively explained by the influence of a changing relative angle between the direction toward the source and the direction perpendicular to the antenna loop. Taking into account the typical speeds of dart stepped leaders on the order of $10^{6} \mathrm{~m} / \mathrm{s}$ [Rakov and Uman, 2003] and the typical duration of the pulse trains $(\sim 200 \mu \mathrm{s})$, the spatial dimension of the underlying lightning phenomena is on the order of a few hundreds of meters. This is by at least 1 order of magnitude lower than the typical distances between the antenna and the thundercloud, which correspond to possible amplitude variations of less than a few per cent within a given train, i.e., significantly lower than the observed variations.

[21] Assuming that the speed of the movement of the dartstepped leader was decreasing with time, we can explain the observed increasing time interval between the neighboring pulses (96\% trains in our data set) on the condition that the distance between the neighboring charge pockets in the thundercloud is nearly constant, forming a hypothetical periodic charge structure at spatial scales on the order of $10 \mathrm{~m}$. We are then also able to explain the sequential decrease of the amplitude of the pulses by the decrease of the speed of the leader propagation, because the radiated magnetic field is proportional to the speed of the leader movement [Uman and McLain, 1970]. Different distances between neighboring charge pockets and/or an increase of the speed of the leader propagation could probably also explain the observed untypical evolution patterns of the trains of the pulses.
[22] Direct balloon measurements have shown that the charge distribution in the thunderclouds is rather complex at larger scales [Stolzenburg and Marshall, 1998]. Extended data set of trains recorded in different conditions is needed to verify the above-mentioned link of temporal properties of the trains to the small-scale properties of the charge structure. Future work using longer recording intervals and simultaneous optical measurements will contribute to the explanation of these fast processes and to their assignment to particular lightning phenomena.

[23] Acknowledgments. This work is an integral part of the cooperation of IAP Prague with LPC2E/CNRS Orleans, France, in the frame of the TARANIS project of CNES. It was supported in part by the international cooperation program of the ASCR (grant M10042120) and in part by GACR 205-09-1253. We are grateful to J. Chum, F. Hruška, J. Souček, J. Vojta, and J. H. Baše for their technical assistance and to P. Novák for comparison of return stroke data with CELDN.

\section{References}

Davis, S. M. (1999), Properties of lightning discharges from multiplestation wide band measurements, dissertation, Grad. Sch. of the Univ. of Fla., UMI Microform 9945961.

Füllekrug, M., and A. C. Fraser-Smith (2011), The Earth's electromagnetic environment, Geophys. Res. Lett., 38, L21807, doi:10.1029/ 2011 GL049572.

Gomes, C., V. Cooray, M. Fernando, R. Montano, and U. Sonnadara (2004), Characteristics of chaotic pulse trains generated by lightning flashes, J. Atmos. Sol. Terr. Phys., 66, 1733-1743.

Krider, E. P., and R. C. Noggle (1974), Broadband antenna system for lightning magnetic fields, J. Appl. Meteorol., 14, 252-256.

Krider, E. P., G. J. Radda, and R. C. Noggle (1975), Regular radiation field pulses produced by intracloud lightning discharges, J. Geophys. Res., 80, 3801-3804.

Krider, E. P., C. D. Weidman, and R. C. Noggle (1977), The electric fields produced by lightning stepped leaders, J. Geophys. Res., 82, 951-960.

Lee, B.-H., D.-C. Jeong, D.-M. Lee, and T. Kawamura (2006), Characteristics of the lightning stepped-leader electromagnetic pulses, Jpn. J. Appl. Phys., $45,933-939$.

Nag, A., and V. A. Rakov (2008), Pulse trains that are characteristic of preliminary breakdown in cloud-to-ground lightning but are not followed by return stroke pulses, J. Geophys. Res. 113, D01102, doi:10.1029/ 2007JD008489.

Nag, A., and V. A. Rakov (2009), Electromagnetic pulses produced by bouncing-wave-type lightning discharges, IEEE Trans. Electromagn. Compat., 51, 466-470.

Rakov, V. A., and M. A. Uman (2003), Lightning-Physics and Effects, Cambridge Univ. Press, New York, ISBN:9780521583275.

Rakov, V. A., R. Thottappillil, and M. A. Uman (1992), Electric field pulses in $K$ and $M$ changes of lightning ground flashes, J. Geophys. Res., 97, 9935-9950.

Rakov, V. A., M. A. Uman, G. R. Hoffman, M. W. Masters, and M. Brook (1996), Burst of pulses in lightning electromagnetic radiation: Observations and implications for lightning test standards, IEEE Trans, Electromagn. Compat., 38, 156-164.

Schonland, B. F. J. (1956), The lightning discharge, Handbuch der Physik, Springer, Berlin, vol. 22pp. 576-627.

Shao, X. M., P. R. Krehbiel, R. J. Thomas, and W. Rison (1995), Radio interferometric observations of cloud-to-ground lightning phenomena in Florida, J. Geophys. Res., 100, 2749-2783.

Stolzenburg, M., and T. C. Marshall (1998), Charged precipitation and electric field in two thunderstorms, J. Geophys. Res., 103, 19777-19790.

Thottappillil, R., V. A. Rakov, and M. A. Uman (1990), $K$ and $M$ changes in close lightning ground flashes in Florida, J. Geophys. Res., 95, 18631-18640.

Uman, M. A., and D. K. McLain (1970), Radiation field and current of the lightning stepped leader, J. Geophys. Res., 75, 1058-1066.

Wang, Y., G. Zhang, T. Zhang, Y. Li, Y. Zhao, T. Zhang, X. Fan, and B. Wu (2010), The regular pulses bursts in electromagnetic radiation from lightning, Asia-Pacific International Symposium on Electromagnetic Compatibility, Beijing, China, doi:10.1109/APEMC.2010.5475814. 